\title{
Paper
}

\section{Spatial Uniformity Improvement at Low Luminance Levels for Organic Light-Emitting Diode Displays}

\author{
Takuya Okada ${ }^{\dagger}$ (member), Takenobu Usui ${ }^{\dagger}$ (member) and Yoshiki Nakajima ${ }^{\dagger}$ (member)
}

\begin{abstract}
This paper proposes two methods for improving uniformity in the low luminance region of an organic light-emitting diode (OLED) display. To supply a stable current to subpixels in the low luminance region, the time-division method produces long-term and short-term subframes for the frame period. On the other hand, the area-division method produces non-emitting and amplified emitting regions of grouped pixels. These methods assume that the effective use of a high current improves spatial uniformity, as it becomes worse in low luminance regions. In experiments involving an actual display, the spatial distribution of luminance was measured while applying each of the proposed methods and evaluated based on the root mean square (RMS) value of the luminous deviation. When the results of the experiments with or without application of the proposed methods were compared, the proposed methods were shown to produce a decrease in the RMS value.
\end{abstract}

Keywords: Display, OLED, Image quality, Mura, Uniformity, Low luminance.

\section{Introduction}

Organic light-emitting diode (OLED) technology has made remarkable progress, providing high-grade displays for television and mobiles, as well as for virtual reality and automotive applications ${ }^{[1-6]}$. As we advance towards a society in which people will have the ability to access important information from anywhere, OLED displays are expected to offer even higher quality and functionality. Today's widely used active-matrix OLED displays are current-driven devices and require a thinfilm transistor (TFT) backplane to control the flow of current to each subpixel. In large-sized displays using oxide TFTs, the spatial uniformity of luminance is a concern, as it affects the image quality of the OLED display. Such uniformity depends on the current supplied to the subpixels in response to the data voltage between the gate and source electrodes of the driving TFT. Since the current responds exponentially to the data voltage, a small variation in voltage is amplified into large variations of luminance.

Several approaches improving the uniformity have been developed. Internal compensation, mainly used for small-sized displays, uses an in-pixel circuit to reset the shifted threshold voltage and provide a stable current to the OLED [7, 8]. External compensation, mainly used for

Received March 15, 2021; Revised June 2, 2021; Accepted July 5, 2021 $†$ NHK Science \& Technology Research Laboratories

(Tokyo, Japan) large-sized displays, is a method whereby an external circuit senses a current or voltage and feeds it back to the digitized signal level [9-11]. Camera compensation, frequently used for the initial calibration of displays, uses a two-dimensional colorimeter to measure the spatial distribution of luminance and adjust the signal levels ${ }^{[12]}$. Pulse-width modulation (PWM) is yet another method for improving uniformity. PWM controls luminance levels by varying the temporal length of the light emission, thus producing more uniform luminance than amplitude modulation, which is commonly used in OLED displays ${ }^{[13,14]}$.

However, despite these developments, the problem of non-uniformity remains, especially in the low luminance region. Figure 1 describes the typical relation between non-uniformity and luminance. As indicated, non-

\section{Non-uniformity}

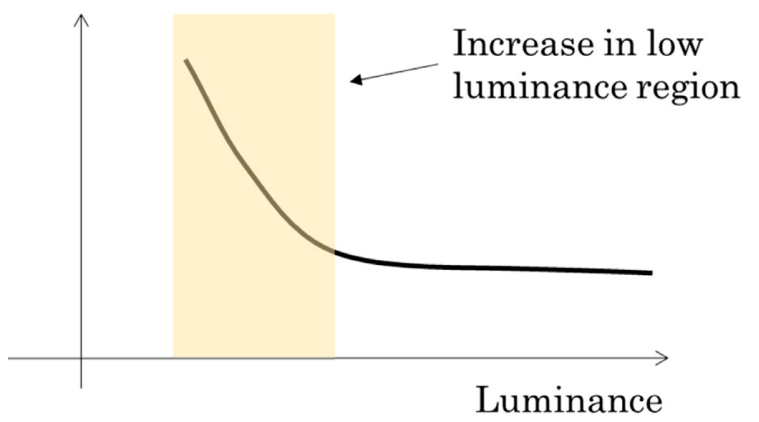

Fig. 1 Typical relation of non-uniformity and luminance levels. 
uniformity increases in the low luminance region, which can contribute to a degradation in an aspect of the image quality such as the contrast ratio. In the low luminance region, the steep subthreshold characteristics of oxide TFTs tend to cause a shift in luminance ${ }^{[15]}$. In highresolution displays that supply minute currents to subpixels, it is difficult to accurately compensate with conventional methods. Therefore, a novel compensation method is needed to improve uniformity at lowluminance levels.

In this study, we propose two different compensation methods: the time-division method and the area-division method. These methods are constructed on the assumption, which non-uniformity increases in the low luminance regions. The time-division method controls the time domain to generate the non-emitting period, while the area-division method controls the spatial domain to generate the non-emitting regions. By dividing the domains into on-emission and off-emission and amplifying the current representing low luminance, a stable current expected to be supplied even in the low luminance region.

\section{Time-division method}

\subsection{Driving scheme}

The time-division method employs two subframes: a short-term subframe and a long-term subframe. Figure 2 shows the driving waveforms of the proposed method for representing low and high luminance levels. The vertical and horizontal axes represent the magnitude of luminescence and time, respectively. For low luminance, (a)

(b)
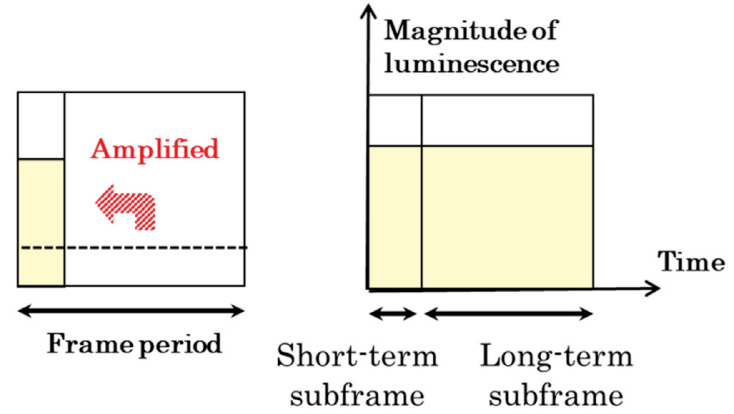

Fig. 2 Driving waveforms during a frame period for representing low (a) and high (b) luminance.

only short-term-subframes are activated, as shown in figure 2 (a). The short-term emissions behave to amplify the current to each subpixel. Figure 2 (a) shows a comparison with the conventional waveform indicated by the dashed line. For high luminance, a long-term subframe is also activated, as shown in figure 2 (b). The amplitude of each subframe is individually controlled to produce the tonal gradation rather than having PWM controlling pulse-width.

Figure 3 shows the signal waveforms on the scan lines and the signal waveform on the data line in timedivision method. The signal on the scan line is transmitted periodically and turns on the switching TFTs sequentially. At this time, the signal on the data line is written into the storage capacitors of the subpixel. Two scans are alternately performed to provide the short-term and long-term subframes. As shown in figure 3 , after Line $\mathrm{k}$ is scanned for the short-term subframe,

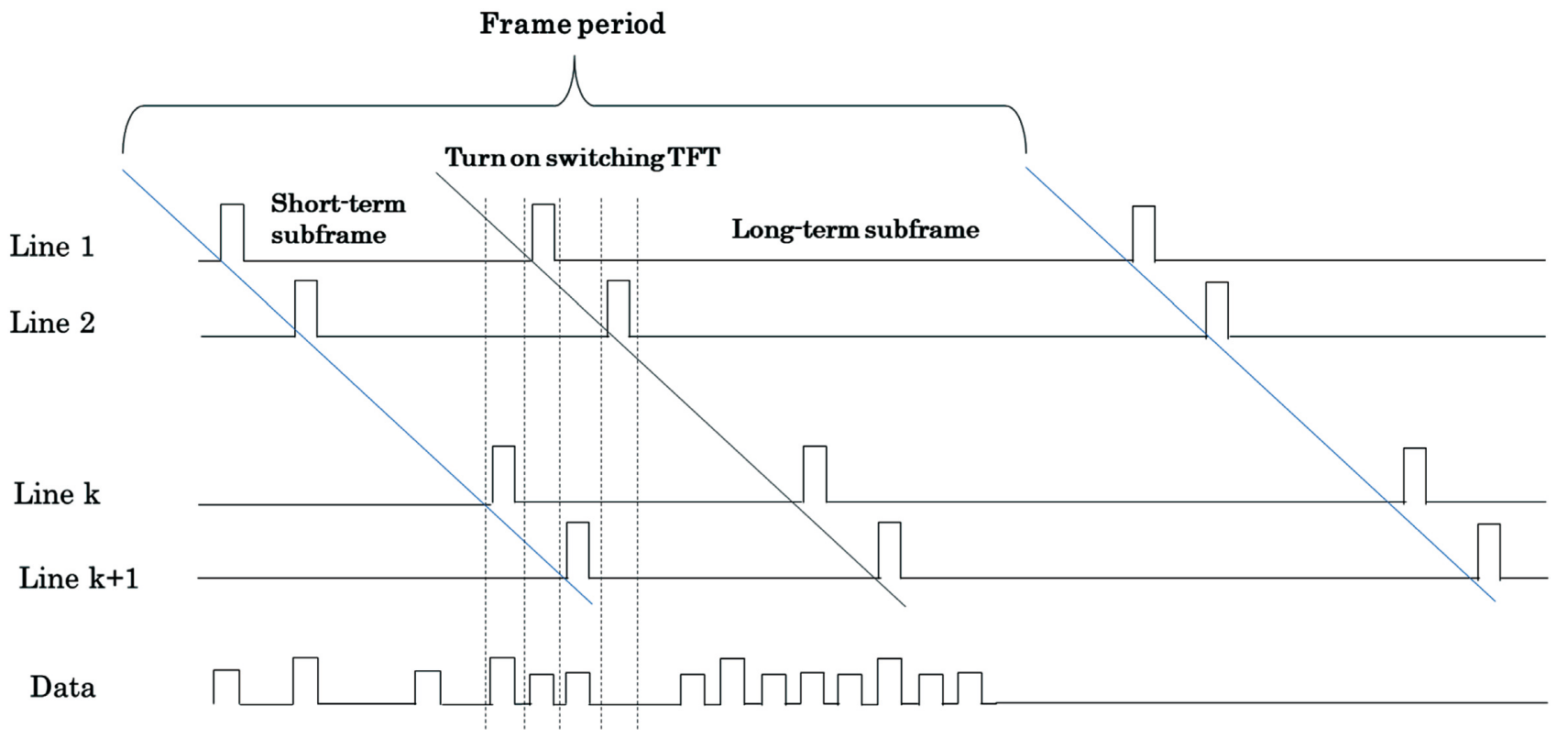

Fig. 3 Data writing sequence for short-term and long-term subframes in time-division method. 


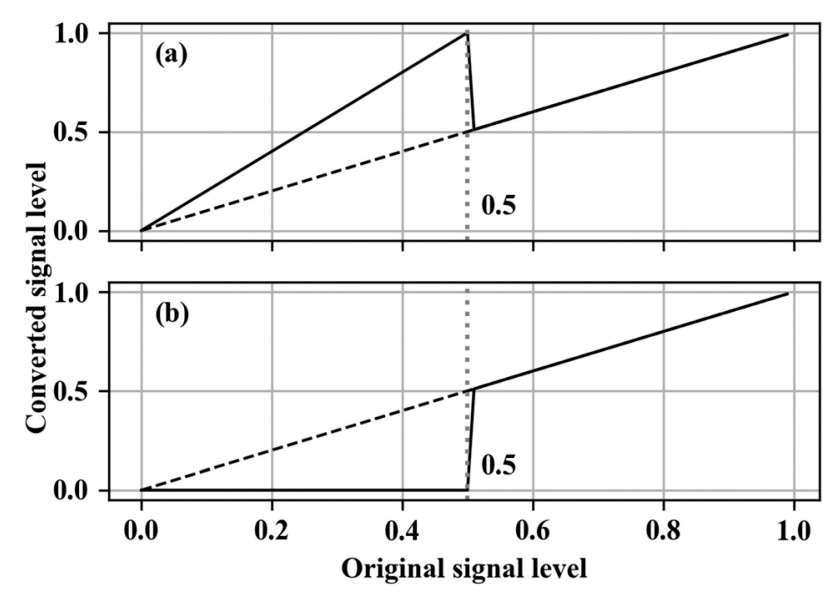

Fig. 4 Examples of a look-up table for short-term (a) and longterm (b) subframes, assuming that the short-term subframe is $1 / 4$ the length of the frame period.

Line 1 is scanned for the long-term subframe. By writing data alternately, the scan speed can be kept within the acceptable range that depends on the characteristics of the switching TFT. In addition, by keeping a duty ratio constant, the structure of the pixel circuit is simplified. On the data lines, the signals need to be transmitted with a speed at least two-fold the frame frequency.

To control the short-term and long-term subframes, a conversion from the original image signal is performed. Figure 4 shows examples of the signal conversion functions for short-term (a) and long-term (b) subframes. In the graphs shown here, the $\mathrm{x}$-axis indicates the original signal level, and the y-axis indicates the converted signal level. The image signal is converted to a signal based on a look-up table so that the emission volume remains constant. If the short-term subframe is $1 / 4$ the length of the frame period, then the signal level is converted approximately two-fold based on the dependence of the luminance level on the signal level. In these examples, the threshold for switching the waveform is set to $1 / 2$. For high luminance, the signal level is converted to satisfy the original correlation shown by the dashed line in figure 4 . For low luminance, the signal level is converted so that the emission in the short-term subframe (a) is amplified and the emission in the long-term subframe (b) is turned off.

\subsection{Experiment using the time-division method}

To validate the proposed subframes, the spatial distribution of luminance was measured using an OLED TV display. The panel size was 65 -inch, and the resolution was $3,840 \times 2,160$. Around $450 \mathrm{~cd} / \mathrm{m}^{2}$ was measured as the maximum luminance when the white level was displayed in a $1 \%$ area of the whole screen. The auto adjustment function of the luminance was

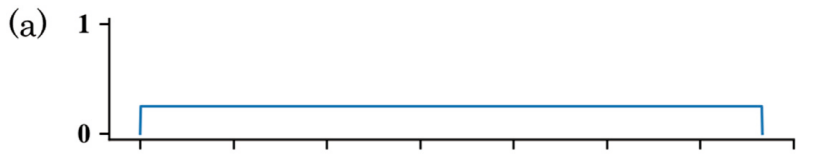

(b)

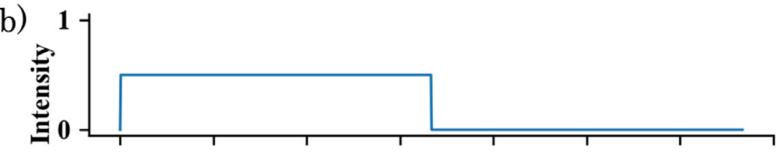

(c)

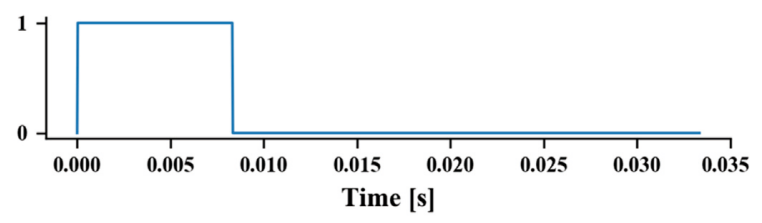

Fig. 5 Driving waveforms with duty ratios of $100 \%$ (a), $50 \%$ (b), and $25 \%$ (c) in experiments with the black image inserted.

turned off. In these experiments, short-term subframes were reproduced by inserting a black image. Figure 5 shows the driving waveforms used in the measurements. In each of the two graphs, the $\mathrm{x}$-axis indicates time, and the y-axis indicates intensity of emission. Figure 5 (a) shows the conventional waveform, while figures 5 (b) and (c) show the waveforms with the black image inserted. Relative to the case described in figure 5 (a), the duty ratios of emission in figures 5 (b) and (c) can be regarded as $50 \%$ and $25 \%$, respectively. Spatial luminance was measured during producing a gray picture with a frame frequency of $30 \mathrm{~Hz}$. As shown in figure 5 , the intensity of emission was adjusted so that the emission volume was constant.

In these experiments, a signal generator and an imaging colorimeter were used for the measurement of the spatial distribution. In the measurement of the imaging colorimeter, the image pixel resolution was $6,572 \times 4,384$. The driving waveforms of figure 5 were produced from a signal generator and the luminance was measured. From experimental results, the deviation from average luminance was evaluated as the luminous deviation $\left(L_{d}\right)$ using

$$
\begin{aligned}
& L_{d}=\frac{L_{i j}-L_{\text {ave }}}{L_{\text {ave }}} \\
& L_{\text {ave }}=\frac{1}{m n} \sum_{i=1}^{m} \sum_{j=1}^{n} L_{i j}
\end{aligned}
$$

where $L_{i j}$ is the luminance of the [i, j]th pixel, and the $(\mathrm{m}, \mathrm{n})$ term represents the numbers of horizontal and vertical pixels extracted from the measurement data, respectively. In the measurement of the imaging colorimeter, the view field was adjusted for the whole 
screen of the OLED display. In addition to this, the average luminance was calculated after the screen area had been accurately extracted from the obtained data. By targeting the whole screen, the uniformity was evaluated the luminance data including all pixels of the OLED display.

\subsection{Results and discussion}

Figure 6 shows the $L_{d}$ histograms produced in the experiments using duty ratios of $100 \%$ (blue line) and $25 \%$ (orange line), respectively. The $x$-axis indicates the luminous deviation, the $y$-axis indicates the frequency of the various deviation levels. The histograms show the uniformity in the low luminance region. In making these measurements, the average luminance was controlled at an equivalent level. As shown, the distribution for the $25 \%$ duty ratio is narrower than that for the $100 \%$ duty ratio.

To compare the luminance deviation, $L_{d}$, for various average luminance levels, root mean square (RMS) values were calculated as

$$
\mathrm{RMS}=\sqrt{\frac{1}{m n} \sum_{i=1}^{m} \sum_{j=1}^{n}\left(\frac{L_{i j}-L_{\text {ave }}}{L_{\text {ave }}}\right)^{2}}
$$

Figure 7 shows the RMS values of $L_{d}$ with average luminance below $5 \mathrm{~cd} / \mathrm{m}^{2}$. The vertical and horizontal axes of the graph represent, respectively, the RMS value and the average luminance. The plotted lines indicate distributions with a duty ratio of $100 \%, 50 \%$, and $25 \%$, and the $25 \%$ estimated distribution from the duty ratio of $100 \%$. As shown, the deviation tends to increase with lower luminance. Notably, the deviations are generally lower in the experiments involving a duty ratio of $25 \%$ as compared to those with a duty ratio of $50 \%$ or $100 \%$.

Given that lager RMS values suggest less uniformity, the $25 \%$ duty ratio distribution with the proposed waveform shows improved uniformity in the low luminance region. As the graph indicates, the uniformity of the $25 \%$ duty ratio distribution appears increasingly superior to that of the $50 \%$ duty ratio distribution as luminance decreases. This result provides evidence that the short-term subframe is effectively involved in the enhancement of uniformity.

Assuming that the RMS value depends on the intensity of emission, the corresponding RMS values can be derived from the relation between the average luminance and the intensity of emission. The red dashed line in figure 7 denotes the $25 \%$ duty ratio distribution estimated from the $100 \%$ duty ratio distribution. Here, the experimental $25 \%$ duty ratio distribution is a little

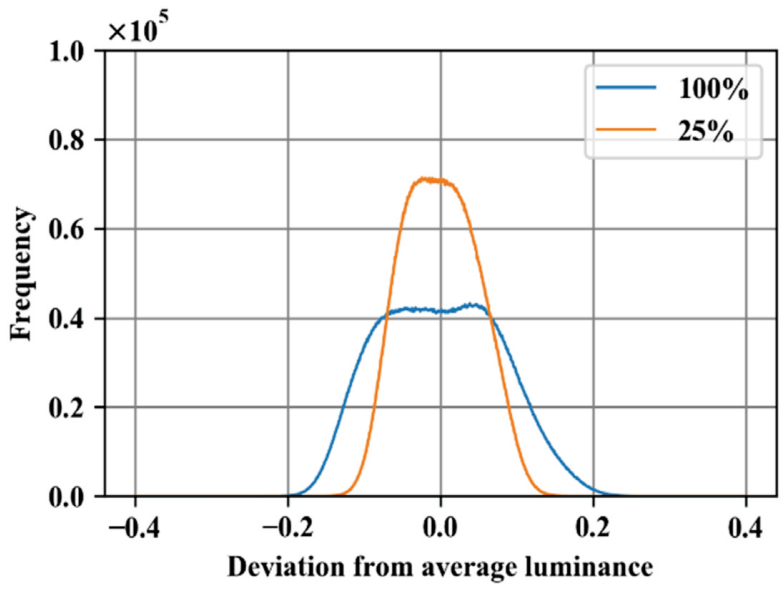

Fig. 6 Histogram of deviation from average luminance in experiments with duty ratios of $25 \%$ and $100 \%$.

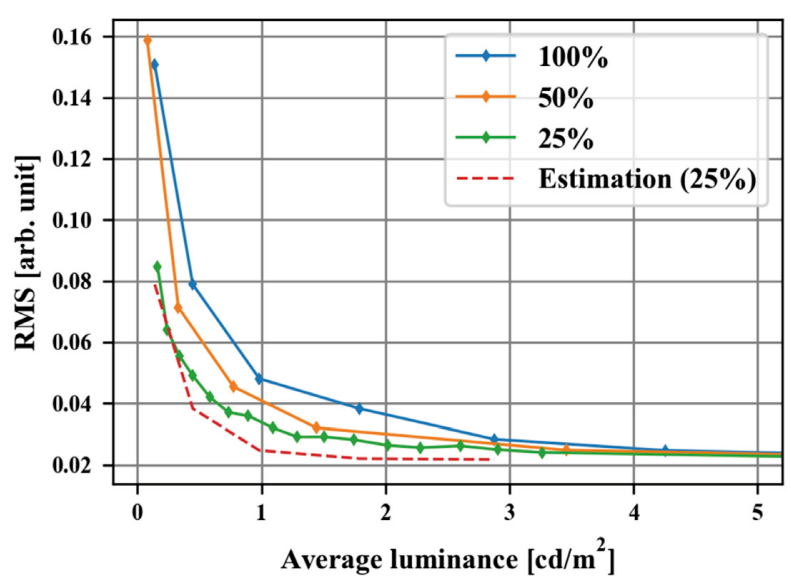

Fig. 7 RMS values of luminous deviations in experiments with duty ratios of $100 \%, 50 \%, 25 \%$, and $25 \%$ estimated values.

worse than the estimated distribution. This result can be attributed to the disagreement of the actual signal levels for the intensity of emission.

The above results demonstrate the effectiveness of using short-term subframes to improve uniformity in low luminance regions. They also indicate the ability of the proposed method to facilitate internal or external compensation techniques by mitigating the deviation to be compensated.

\section{Area-division method}

\subsection{Driving scheme}

The area-division method, another approach to compensate non-uniformity, produces two spatial regions: a non-emitting region and an amplified emitting region. Figure 8 illustrates each of two regions. With the areadivision method, four pixels are divided into two groups; one group emits in the first frame and the other group emits in the second frame. The luminance in the emitting 


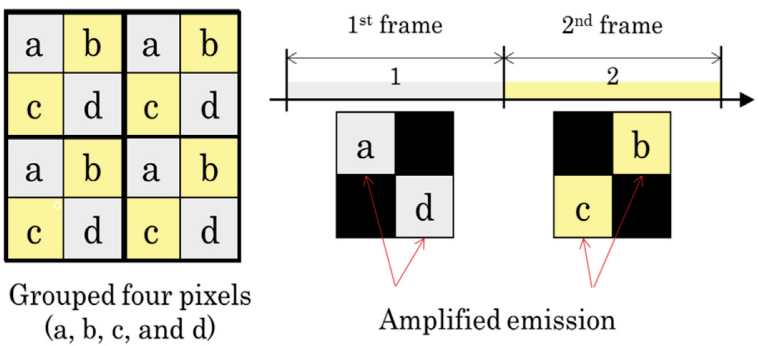

Fig. 8 Non-emitting region and amplified emitting region in the area-division method.

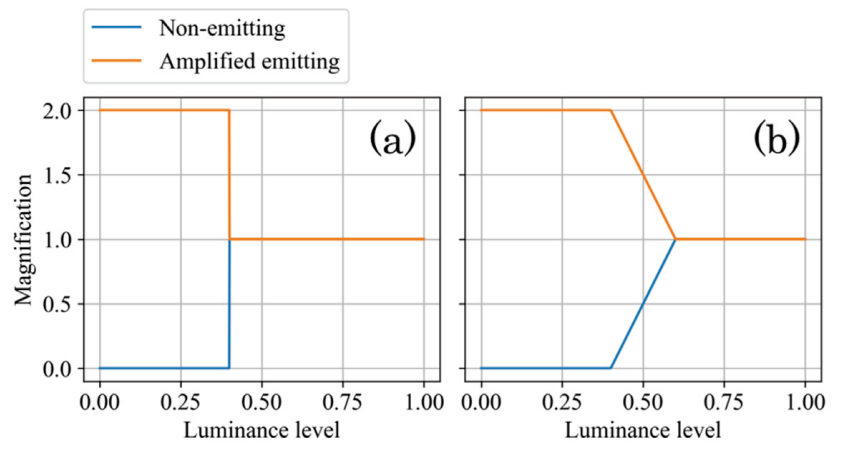

Fig. 9 Control functions for luminance and amplified emitting region in the area-division method.

region is amplified two-fold, and the light is alternately emitted between the two frames to equalize the luminance. The low signal values are converted into zero or two-fold to compensate non-uniformity in the low luminance region. In a high-resolution display, the nonemitting region and the amplified region are perceptually integrated and perceived as a continual image.

Figure 9 shows the control functions for luminance in the non-emitting and the amplified emitting regions. The luminance is amplified two-fold in the amplified emitting region and turned off in the non-emitting region. Figure 9 (a) shows the function that luminance changes discontinuously at the luminance level of 0.4 . Such function is possible to deteriorate images if the signal values more and less than the threshold are adjacent. To address this problem, we propose the function including the buffering region that the luminance is smoothly connected in the intermediate luminance region from 0.4 to 0.6 , as shown in figure 9 (b). This buffers the large variations of luminance and improves our area-division method.

\subsection{Experiment using the area-division method}

An experiment was conducted in which the areadivision method was applied to gray image signals from a signal generator. The spatial distribution of luminance was measured using a two-dimensional colorimeter in an OLED display. The used OLED display was the same

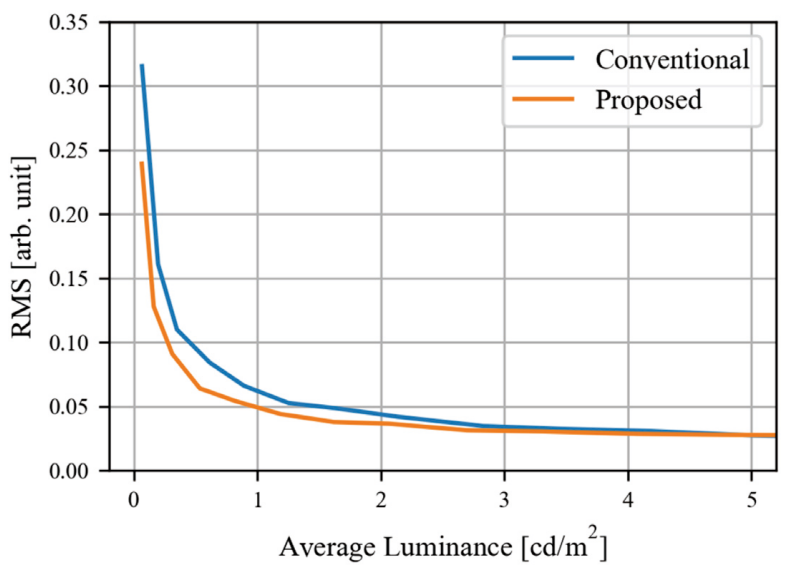

Fig. 10 RMS values of luminous deviations in experiments with the proposed area-division and the conventional method.

as section 2. The deviation from average luminance was evaluated according to equation (1), the deviation using the area-division method was compared to the conventional deviation based on RMS value calculated according to equation (3).

\subsection{Results and discussion}

Figure 10 shows the RMS values for the area-division method and the conventional method. As shown, the RMS values are lower with the area-division method in the low luminance region. Since the distance from the colorimeter to the display in this experiment differs from the experiment involving the time-division method, the RMS values are unrelated to these in figure 7. As shown in figure 10, when the conventional distribution is compared with the proposed method, an improvement of uniformity is evident in the low luminance region. Above results demonstrate the effectiveness of the proposed area-division method to improve uniformity in the low luminance regions.

\section{Conclusion}

We conducted experiments to validate the effectiveness of the proposed two methods. In experiments involving the time-division method, an improvement in uniformity was demonstrated from the spatial measurement of luminance, when the short-term subframe was reproduced with duty ratios of $25 \%$ and $50 \%$. The $25 \%$ duty ratio distribution showed better uniformity than the $50 \%$ duty ratio. In a comparison of the experimental and the estimated distributions, the experimental $25 \%$ duty ratio distribution was slightly worse than the estimated distribution. This result can be attributed to the disagreement of the actual signal levels for the intensity of emission. In experiments involving the proposed area- 
division method, an improvement in uniformity was also demonstrated. These results illustrate the potential of the two proposed methods to improve uniformity in low luminance regions for OLED displays.

\section{References}

1) H. Shin, K. Park, S. Takasugi, Y. Jeong, J. Kim and C. Oh, "A highimage-quality OLED display for large-size and premium TVs", SID DIGEST, 48, pp.1134-1137 (2017)

2) Z. Wu, L. Yan, Y. Li, X. Feng, H. Shih, T. Kim, Y. Peng, J. Yu and X. Dong, "Development of 55-in. 8K AMOLED TV based on coplanar oxide thin-film transistors and inkjet printing process", J. SID, Vol. 28, No. 5, pp.418-427 (2020)

3) Z. Yu, Z. Wang, G. Liu, B. Wang, P. Jang, D. Wang and H. Qiu, "1000PPI LTPS OLED display for VR application", SID DIGEST, 12-4, pp.156-159 (2020)

4) J. Lee, T.T. Nguyen, J. Bae, G. Jo, Y. Lee, S. Yang, H. Chu, J. Kwag, "5.8-inch QHD flexible AMOLED display with enhanced bendability of LTPS TFTs", J. SID, Vol. 26, No. 4, pp.200-207 (2018)

5) Y. Takeda, M. Aman, S. Murashige, K. Ito, I. Ishida, H. Matsukizono and N. Makita, "Automotive OLED display with high mobility top gate IGZO TFT backplane", ITE Trans. on MTA Vol. 8, No. 4, pp.224-229 (2020)

6) X. Mou, L. Xiang, G. Wang, X. Huang, C. Liang, G. Chen, L. Zhang, S. Li, X. He, W. Hu, K. Yang, Z. Yu, Y. Yang, Y. Feng, D. Wang, H. Qiu, W. Gao, "High reliability flexible AMOLED display with algorithm compensation for automotive application", SID DIGEST, 11-1, pp.130-133 (2020)

7) C. Lin, P. Lai, L. Shih, C. Hung, P. Lai, T. Lin, K. Liu and T. Wang, "Compensation pixel circuit to improve image quality for mobile AMOLED", IEEE J. Solid-state Circuits, Vol. 54, No. 2, pp.489-500 (2019)

8) Y. Jang, D. Kim, W. Choi, M. Kang, K. Chun, J. Jeon, Y. Ko, U. Choi, S. Lee, J. Bae, K. Park, S. Yoon and I. Kang, "Internal compensation type OLED display using high mobility oxide TFT", SID DIGEST, 7-4, pp.76-79 (2017)

9) S. Takasugi, H. Shin, M. Chang, S. Ko, H. Park, J. Lee, H. Kim and C. Oh, "Advanced compensation technologies for large-sized UHD OLED TVs", J. SID, Vol. 24, No. 7, pp.410-418 (2016)

10) B. Huang, J. Fan, X. Xu and X. Guo, "Displaying-synchronous open-loop external compensation for active-matrix light emitting diode displays", IEEE Trans. Circuit and Systems, Vol. 67, No. 10, pp.1790-1794 (2020)

11) L. Verschueren, M. Ameys, M.V. Lopez, S. Smout, T.H. Ke, E. Vandenplas, A.J. Kronemeijer, P. Heremans, J. Genoe, W. Dehaene and K. Myny, "A 2T1C AMOLED display with external compensation reducing on-panel current variations to $0.079 \% "$, SID DIGEST, 38-4, pp.547-550 (2020)
12) H. Shin and T. Kim, "Enhancement of the luminance uniformity in large-size organic light-emitting devices based on In-Ga-Zn-O thinfilm transistors by using a new compensation method", J. Elect. Dev. Soc., Vol. 7, pp.557-560 (2019)

13) M. Kimura, S. Sawamura, M. Kato, Y. Hara, D. Suzuki, H. Hara and S. Inoue, "Pulse-width modulation with current uniformization for TFT-OLEDs", IEICE Trans. Elec., E90-C, 11, pp.2076-2082 (2007)

14) P. Volkert, X. Jiang and C. Xu, "Characterization and compensation of OLED aging in a digital system", J. SID, Vol. 23, No. 12, pp.570-579 (2015)

15) T. Nishiyama, K. Tanaka, K. Yamamoto, K. Yamamoto, S. Uchida, R. Yonebayashi, T. Miyamoto, M. Hosokawa and H. Katoh, "Pixel circuit with backgate feedback for deep and rich black expressions on lightemitting display", SID 2019 DIGEST, 31, pp.1329-1332 (2019)

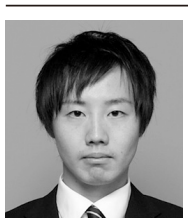

Takuya Okada received his B. Eng. and M Eng. degrees from University of Tsukuba, Ibaraki, Japan, in 2014 and 2016, respectively. In 2016, he joined the Science \& Technology Research Laboratories of Japan Broadcasting Corporation (NHK), Tokyo, and has been researching on the signal processing and the driving technology of displays.

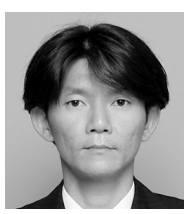

Takenobu Usui received his B. Eng. and M. Eng. degrees from Tokyo Institute of Technology, Tokyo, Japan, in 2002 and 2004, respectively. In 2004, he joined Japan Broadcasting Corporation (NHK). Since 2007, he has been at the Science \& Technology Research Laboratories of NHK. He is currently working on the signal processing and the driving technology of displays.

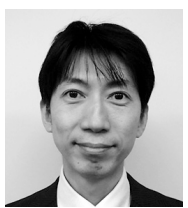

Yoshiki Nakajima received the B. Eng., M. Eng., and Dr. Eng. degrees in electrical and electronic engineering from Tokyo University of Agriculture and Technology, Tokyo, Japan, in 2000, 2002 and 2004, respectively. In 2004, he joined Japan Broadcasting Corporation (NHK), Tokyo, and worked at the Broadcast Engineering Department of NHK Broadcasting Center. Since 2005, he has been at the Science and Technology Research Laboratories of NHK. He is currently working on the development of flexible OLED displays. 